Cinémas

Revue d'études cinématographiques

Journal of Film Studies

\title{
La critique et la disparition de son objet
}

\section{Gilles Thérien}

Volume 6, numéro 2-3, printemps 1996

\section{La Critique cinématographique}

URI : https://id.erudit.org/iderudit/1000977ar

DOI : https://doi.org/10.7202/1000977ar

Aller au sommaire du numéro

\section{Éditeur(s)}

Cinémas

\section{ISSN}

1181-6945 (imprimé)

1705-6500 (numérique)

Découvrir la revue

\section{Citer cet article}

Thérien, G. (1996). La critique et la disparition de son objet. Cinémas, 6(2-3), 141-163. https://doi.org/10.7202/1000977ar

\section{Résumé de l'article}

La critique cinématographique a fort à faire lorsqu'il lui faut parler du cinéma actuel. Au plan de la technique, on ne produit plus de mauvais films et l'objet-film a une tendance à devenir neutre au plan esthétique. Il doit s'adapter à trop de normes, à trop de médias de diffusion. Il ne reste plus que l'histoire, le récit qu'il faut critiquer sans le dévoiler. Les grandes maisons de production accompagnent leur diffusion de dossiers étoffés sur les films qui fournissent à la critique tout ce qu'il faut savoir sur le produit sans avoir à faire de recherche. La critique publique est condamnée à l'inefficacité et elle doit chercher du côté de la critique spécialisée, universitaire ou non, un complément de savoir. Or, même la critique universitaire est obnubilée par les procédés narratifs. Aussi faut-il consentir un effort particulier pour redonner à la critique un rôle, une fonction à l'endroit du cinéma. La question est discutée à travers l'analyse d'un cas particulier, mais exemplaire, Schindler's List de Steven Spielberg. C'est à travers une forme de variation critique que ce film peut éveiller chez le critique et le spectateur un jugement qui va au-delà de l'histoire racontée. Il y retrouve alors un film inquiétant, étrange malgré les prix et les distinctions dont il a été inondé.
Ce document est protégé par la loi sur le droit d'auteur. L’utilisation des services d'Érudit (y compris la reproduction) est assujettie à sa politique d'utilisation que vous pouvez consulter en ligne.

https://apropos.erudit.org/fr/usagers/politique-dutilisation/ 


\title{
La critique et la disparition de son objet
}

\section{Gilles Thérien}

\begin{abstract}
RÉSUMÉ
La critique cinématographique a fort à faire lorsqu'il lui faut parler du cinéma actuel. Au plan de la technique, on ne produit plus de mauvais films et l'objet-film a une tendance à devenir neutre au plan esthétique. Il doit s'adapter à trop de normes, à trop de médias de diffusion. Il ne reste plus que l'histoire, le récit qu'il faut critiquer sans le dévoiler. Les grandes maisons de production accompagnent leur diffusion de dossiers étoffés sur les films qui fournissent à la critique tout ce qu'il faut savoir sur le produit sans avoir à faire de recherche. La critique publique est condamnée à l'inefficacité et elle doit chercher du côté de la critique spécialisée, universitaire ou non, un complément de savoir. Or, même la critique universitaire est obnubilée par les procédés narratifs. Aussi faut-il consentir un effort particulier pour redonner à la critique un rôle, une fonction à l'endroit du cinéma. La question est discutée à travers l'analyse d'un cas particulier, mais exemplaire, Schindler's List de Steven Spielberg. C'est à travers une forme de variation critique que ce film peut éveiller chez le critique et le spectateur un jugement qui va audelà de l'histoire racontée. Il y retrouve alors un film inquiétant, étrange malgré les prix et les distinctions dont il a été inondé.
\end{abstract}

\section{ABSTRACT}

Film criticism faces a heavy task when it comes to the discussion of current cinema. On the technical level, there are no longer any badly made films, and the filmic object has a tendency to become neutral on the 
esthetic level. It must now adapt itself to too many norms, to too many forms of diffusion. All that remains is the story, the plot, which must be criticized without being revealed. The big production companies send out thoroughly documented press kits on their films, which supply the critic with everything there is to know about the product so that there is no need for research. Public criticism is condemned to inefficiency and has to look to specialized criticism, academic or otherwise, for additional information. But even academic criticism is clouded by narrative procedures. Should we therefore undertake a special effort to restore to criticism its role, its function with respect to cinema? This issue is discussed in an analysis of one particular, exemplary case, Steven Spielberg's Schindler's List. By means of a form of critical variation, this film succeeds in awakening in both the critic and the spectator a judgment that goes beyond the story it tells. It is thus a disturbing film, alien despite the many prizes and honours with which it was crowned.

Commençons par une bonne nouvelle: le mauvais cinéma n'existe plus... du moins, presque plus. Ce n'est pas une boutade. Il s'agit d'une constatation qui tient à la nature même du nouveau cinéma qui a supplanté l'ancien. Je ne sais trop à quelle date cela est arrivé. Les historiens pourront peut-être un jour nous la préciser, mais je connais la constellation d'éléments qui font que le mauvais cinéma a disparu. Il existe, on s'en doute bien, ici et là, quelques "mauvais" films, mais ce sont des accidents de la nouvelle "nature" cinématographique. À bien y regarder, l'objet-cinéma, lui, peut être tout à fait impeccable.

Les modes de production du cinéma actuel ne permettent plus l'existence d'un film mal fait. Ce qui, il y a quelques années, était encore possible par je ne sais quelle gaucherie d'apprentissage ou par insuffisance technique, a disparu grâce à la standardisation des techniques professionnelles de production. Un film coûte suffisamment cher pour qu'on s'assure que le résultat matériel soit impeccable. Cette standardisation se fait dans l'ombre des studios. Un film est un produit que l'on tourne de façon économique, c'est-à-dire sans égards pour les exigences esthétiques que le tournage lui-même pourrait engendrer. Il faut dire que bien 
peu de réalisateurs ont le loisir de tourner ce qu'ils veulent et de la façon qu'ils le souhaitent. L'organisation du tournage se fait en fonction des impératifs matériels de la production, en gros des coûts qu'il faut contrôler par une utilisation économique et rationnelle de l'équipe technique, des lieux de tournage, des comédiens et comédiennes qui, dans la plupart des cas, coûtent moins cher si on les emploie moins longtemps. Il est bien rarement question de faire des grands choix esthétiques en prenant une pellicule Agfa plutôt qu'une pellicule Eastman pour modifier l'importance des bleus de cette dernière au profit des verts de la première. De toutes les façons, plus personne ne remarque la couleur des films, soit parce qu'ils les voient à la télévision et c'est la définition numérique des couleurs qui prime - elle est même devenue automatique sur plusieurs postes —, soit parce que le registre personnel de perception des couleurs du spectateur s'est appauvri à cause de la standardisation vidéo des couleurs. Et nous n'avons pas parlé des effets spéciaux qui peuvent créer des scènes entières dans des films comme Who framed Roger Rabbit ou Jurassic Park, scènes que le spectateur moyen a parfois beaucoup de difficulté à identifier comme étant "spéciales". L'effet spécial est de plus en plus une façon de mettre du "réalisme" dans les films. C'est cette même technique, d'ailleurs, qui permet de corriger à peu près n'importe quelle erreur dans une séquence de façon à masquer ce qui pourrait apparaître comme un défaut de l'objet-cinéma. Il est difficile aujourd'hui de ne pas avoir le sentiment qu'avec les nouvelles techniques et la standardisation de la production, le cinéma a atteint sa maturité médiatique. Il apparaît de plus en plus comme un instrument de musique dont on a développé jusqu'à la limite la gamme des possibilités, un peu comme des orgues qui vont des plus petites aux plus grandes tout en restant identiques les unes aux autres. Bref, le cinéma ne se donne plus facilement à juger sous l'angle de sa matérialité. Le film que je vois n'est pas un objet d'art dont je peux apprécier les qualités propres '. Sa mise en marché en tient d'ailleurs compte. On multiplie les moyens pour créer autour d'un film une attente qui est proportionnelle aux profits escomptés. La publicité d'un film commence bien avant sa mise en production et, surtout, bien avant sa diffusion. Les journalistes sont invités au tournage, 
les vedettes donnent des entrevues, le réalisateur s'explique sur son futur chef-d'œuvre dans une sorte de mouvement croissant qui atteint son sommet à la sortie du film, le même jour, en " $\mathrm{x}$ " copies, dans " $\mathrm{y}$ " villes, de " $\mathrm{z}$ " pays. Nous atteignons alors le degré zéro de la consommation passive et le discours sur le film vient s'insérer dans le battage publicitaire général où même une mauvaise critique sert la cause du film surtout si elle vient d'un média important.

Au moment où la réalité virtuelle commence à prendre de plus en plus de place, au moment aussi où le contact avec l'ordinateur engage une approche de plus en plus interactive des réseaux d'images, de textes et de sons, on peut se demander de quoi sera fait l'avenir du film et du cinéma. Allons-nous vers une forme de divertissement hautement codée comme le cirque où il sera difficile de retracer des inventions personnelles, ou allons-nous vers une standardisation du récit en images raconté pour satisfaire tel ou tel groupe bien ciblé d'individus? Le cinéma chez soi avec grand écran, haute définition et son surround $^{2}$ est en train d'achever la fermeture du média sur des objectifs précis: le loisir, le désennui, le "remplissage" des périodes creuses. L'esprit critique qui a permis au cinéma d'évoluer ne sera plus nécessaire. Or, c'est ce dernier qui commence le plus sérieusement à faire défaut. Cela se comprend d'autant plus facilement que la critique est elle-même en panne.

Face aux divers changements que nous venons d'indiquer, la critique cinématographique éprouve un sérieux malaise que nous allons tenter d'illustrer sans toutefois pouvoir y apporter quelque remède que ce soit. La situation semble bloquée et on ne peut qu'espérer un esprit d'innovation encore lent à se manifester. $\mathrm{La}$ critique publique traditionnelle est au cœur d'un changement profond qui l'éloigne progressivement des autres formes de critique: la critique spécialisée et la critique universitaire.

La critique publique, ou d'intérêt public, est celle qui s'adresse au consommateur de films. Elle accompagne la sortie de certains films dans les journaux, à la télévision, à la radio. Au cours des dernières années, elle est devenue multiforme, à l'image du marketing qui la téléprogramme. En effet, cette critique commence parfois au début d'un tournage et comprend 
un accueil des divers médias sur les lieux de tournage, des rencontres avec les vedettes, un résumé du scénario, c'est-à-dire les grands segments de l' "histoire" situés dans un cadre générique précis, un film policier, un film de science-fiction, etc. S'il s'agit d'un film québécois, on insistera sur le réalisateur, sur ses autres films et sur les vedettes que tous connaissent. En fait, la critique publique se met en marche alors que le produit n'existe pas encore, qu'il n'en est encore qu'à l'étape de projet. Les producteurs habiles laissent " couler " diverses informations susceptibles d'intéresser les médias et ce battage publicitaire auquel tous consentent volontiers engage déjà le regard critique. Au Québec, cela vaut tout autant pour le téléfilm que pour le cinéma tourné pour la diffusion en salle. Quand il s'agit de films étrangers, et je pense ici surtout aux films hollywoodiens, leur diffusion en salle s'accompagne d'un battage publicitaire orchestré par les distributeurs, battage qui comprend des dossiers de presse sur le film. On met à la disposition du critique œuvrant dans divers médias un matériau tout prêt qui lui permettrait, le cas échéant, de faire la critique d'un film sans même l'avoir vu.

En fait, on cherche une seule chose: rejoindre le consommateur pour que ce dernier aille voir le film en salle, se branche sur la télé ou loue la cassette vidéo du film. Dans l'état actuel des choses, aucun média n'est en mesure de dire non à cet assaut qui se traduira évidemment par un gain, un profit dans la longue chaîne qui tient ensemble producteurs de cinéma, distributeurs, journaux, réseaux de télévision ou de radio. On voit mal comment un journal, par exemple, pourrait affirmer une quelconque indépendance d'esprit vis-à-vis ceux qui font vivre leurs sections "spectacles" ou "cinéma». Le consommateur, le cinéphile ont bien de la difficulté à retrouver une vision critique dans tout cela. Ainsi, on reconnaissait récemment que, malgré des critiques cinématographiques plutôt mauvaises au moment de la sortie du film Waterworld de Kevin Costner, la production la plus dispendieuse à ce jour du cinéma, ce dernier était en train de faire de l'argent grâce, justement, au fait qu'on continuait d'en parler, qu'on encourageait la polémique, que les droits de télévision et les droits de vente et de location de cassettes laissaient anticiper un succès économique. 
De toutes les façons, dans la perspective d'une culture hollywoodienne, les consommateurs ont appris à désirer d'abord et avant tout une bonne histoire, de l'aventure et de l'action, des effets spéciaux éblouissants et un son qu'envient les discothèques. On (re)verra un film à la télévision dans de piètres conditions techniques, "reformaté" pour l'écran télévisuel quand il n'a pas tout simplement subi des altérations de montage pour diverses fins. De toutes les façons, il sera habituellement, dans les chaînes non spécialisées, charcuté au profit d'une publicité qui brise la concentration du spectateur tout en fragmentant le film. L'objet-cinéma a beaucoup de difficulté à garder son unité.

Pour consterner un peu plus le lecteur si cela était encore nécessaire, je me suis amusé à relever les films présentés à la télévision pendant une semaine. La "critique" de ces films est réduite au minimum, une cote numérique de 1 à $7^{3}$. Le 1 indique évidemment qu'il s'agit d'un chef-d'œuvre, le 2, d'un film remarquable, le 3 d'un très bon film, le 4 d'un bon film, le 5 d'un film moyen, le 6 d'un film pauvre alors que le 7 est réservé au film jugé minable. Dans une semaine de cinéma-télé, on a présenté, pour la grande région de Montréal, 182 films dont 31 "premières mondiales", en général des téléfilms qui ne recevront de cote qu'à leur prochaine diffusion. Parmi les 151 autres films, soit $83 \%$ des films diffusés, un seul portait la cote 1, 8 la cote 2 et 20 la cote 3 . Dans ces deux derniers cas, 6 sur les 8 films qui portaient la cote 2 n'étaient accessibles que sur abonnement spécial, et le quart des films cotés 3 était dans la même situation. 73 films portaient la cote 4 , soit à peu près la moitié des 151, 38 films étaient cotés 5 et 11, 6. C'est dire la mauvaise qualité des films diffusés. Qu'il n'y ait eu aucun film coté 7 ne change rien au fait que 49 films se partageaient la catégorie "moyen" et "pauvre" contre 29 dans les trois meilleures catégories. On pourrait évidemment discuter de la valeur des cotes, mais l'ensemble de la situation représente une courbe en forme de cloche avec une prépondérance de sa surface négative ce qui, en docimologie, indique un ensemble plutôt faible. Cet exercice n'a qu'un but: insister sur le fait que la télévision ne cherche pas à remplacer les anciens cinémas d'art et d'essai ou la cinémathèque. D'ailleurs, illustration probante, le seul film classé comme 
chef-d'œuvre était diffusé à PBS. On le voit, le consommateur ordinaire et le cinéphile sont aussi mal servis l'un que l'autre par la télévision. On ne trouve pas beaucoup d'éléments qui leur permettraient de se former un esprit critique ou même une culture cinématographique. La télévision comme média de diffusion du cinéma et la location de vidéocassettes sont à la remorque des modalités de la diffusion en salle. On s'habitue à regarder des films esthétiquement pauvres et on ne parvient plus à exercer son esprit critique face à l'objet lui-même: la narration prend toute la place.

Ce qui est dénoncé ici ne l'est jamais par la critique publique ou, si cela est, c'est parce que tous sont du même avis, ce qui en diminue d'autant le risque. L'exercice critique s'est fragmenté, ponctualisé. En regardant les critiques de certains films, je lis un résumé de leur "histoire" avec les obligatoires "je-ne-vousrévélerai-pas-la-fin». Au mieux, je trouve un renforcement du star system lorsqu'on parle du jeu de telle ou telle vedette; rarement d'ailleurs parle-t-on du personnage. Le film et son "histoire" sont une seule et même chose dans l'esprit de bien des critiques. Il faut dire au lecteur quelle histoire mérite son attention.

Il existe une autre critique, celle que je dirai spécialisée. C'est celle que l'on retrouve dans les revues consacrées au cinéma et qui se permettent de suivre les événements cinématographiques un peu partout de par le monde: festivals locaux, séances spéciales, le cinéma de répertoire ou encore les films marginaux. Il reste encore de ces revues, mais il en reste de moins en moins parce que, si elles ne sont pas supportées par l'État ou la profession, elles ne peuvent survivre en toute liberté face au marché du cinéma qui, en général, n’a que faire de ce type de publication qui ne suit pas la vague du marché de diffusion. Il est possible, dans les pages de ces revues, de se donner au moins un début de formation à la culture cinématographique, ne serait-ce que parce que l'on trouve là des titres de films, des noms de réalisateurs, des points de vue sur l'évolution du média qu'on ne peut retrouver ailleurs. Elles sont un complément aux quelques rares lieux de location de cassettes d'un cinéma inscrit dans le temps, dans l'histoire comme dans les ethnies. Elles recoupent 
parfois ce que je nommerai plus loin la critique universitaire, elles recoupent aussi volontiers et avec une connaissance plus pointue, la critique publique mais, achronique, elle n'a aucune influence sur le consommateur et encore moins sur le producteur.

Ce que je nomme critique universitaire est, de fait, le résultat du développement des études cinématographiques. Ces dernières sont justement là pour favoriser le développement de l'esprit critique en mettant en valeur les différents aspects du cinéma tant sur le plan technique que sur le plan esthétique. Les études cinématographiques n'ont pas, me semble-t-il, à montrer comment faire du cinéma, mais comment il se fait. L'étude comme la pratique de la technique devraient avoir d'abord une fonction de formation. Dans ce cadre, l'élément important est la place d'une culture cinématographique globale qui propose comme un savoir incontournable l'histoire du cinéma, le progrès de ses techniques, les moments forts de son évolution, l'esthétique de ses auteurs, la mise en question de la nature du cinéma. Évidemment, on ne peut pas demander à la critique universitaire de se substituer à la critique publique, cela n'est pas de son ressort. Elle n'en a ni les moyens ni la capacité comme institution. Elle est trop lente et trop spécialisée. On attend donc d'elle un discours qui nous sorte du quotidien, des modes, de l'emprise de la consommation. Elle est là pour nous faire réfléchir sur le média lui-même, sur sa nature et son évolution. Pourtant, dans les dernières années, par un curieux hasard, la critique universitaire s'est intéressée de façon très importante, dans la foulée des travaux structuralistes et narratologiques menés en littérature, à l'aspect "récit" des films, délaissant très souvent l'aspect matériel du film au profit de sa diégèse. En cela, la critique universitaire condamne celui qui la suit à se retrouver devant le même problème que lui pose la critique publique. Il ne sait plus que faire des éléments techniques d'un film: photographie, montage, travail sur les formes, relations entre les séquences, esthétique développée sur plusieurs plans, toutes choses qui ont fait partie du regard de la critique traditionnelle. La critique universitaire actuelle lui donne un moyen plus "scientifique" de gérer la narration d'un film. À la limite, elle fait de façon un peu plus 
lourde ce qu'un critique intuitif peut faire légèrement et facilement. Cette tendance se trouve combattue soit par un retour aux études traditionnelles historiques, soit par un recours aux études d'inspiration culturelle (cultural studies) où l'on retrouve divers amalgames qui vont de la déconstruction au postmodernisme en passant par la psychanalyse ou les études sur le gender. $\mathrm{Si}$ la critique universitaire se réoriente, ce pourrait être en fonction de la constitution d'un monde imaginaire qui naît dans les films comme dans les autres médias visuels et qui cherche à s'harmoniser avec l'imaginaire individuel ou social des spectateurs. La critique universitaire se doit d'explorer les frontières du média cinéma en refusant de se laisser piéger par des données qui réduisent le film à son seul contenu narratif que des images ont le devoir d'illustrer.

Chaque film, selon son importance, met en jeu la critique, toute la critique. Comment concilier alors les diverses critiques de façon à ne pas se retrouver constamment renvoyés dans la marge parce que nos jugements sont de l'ordre de la répétition du schéma présenté par les diffuseurs ou exprimés dans un délai qui en disqualifie la pertinence? Il me semble intéressant de mettre ces diverses questions à l'épreuve à partir de films qui ont connu du succès, voire une immense popularité, tout en présentant des problèmes particulièrement difficiles aux plans formel et esthétique comme au plan narratif. J'ai choisi Schindler's List de Steven Spielberg, produit en 1993 à partir d'un roman du même nom de Thomas Keneally. Le film a été à peu près universellement bien accueilli comme le roman d'ailleurs. Il s'est mérité de nombreux prix et a permis à la représentation de l'Holocauste de s'arrimer de façon spectaculaire à l'univers hollywoodien. Mon questionnement portera sur le sujet lui-même, son traitement esthétique et, d'une certaine façon aussi, sur certains éléments de sa réception.

En 1993, au moment de sa diffusion canadienne, Schindler's List est accompagné, comme il se doit pour un film de Spielberg, d'un dossier de presse qui comporte les éléments suivants: une série de photographies, un document technique qui reproduit le générique du film et un document qui raconte à la fois l'histoire de la production de Schindler's List de même que celle de son contenu. Le document se termine par des notices 


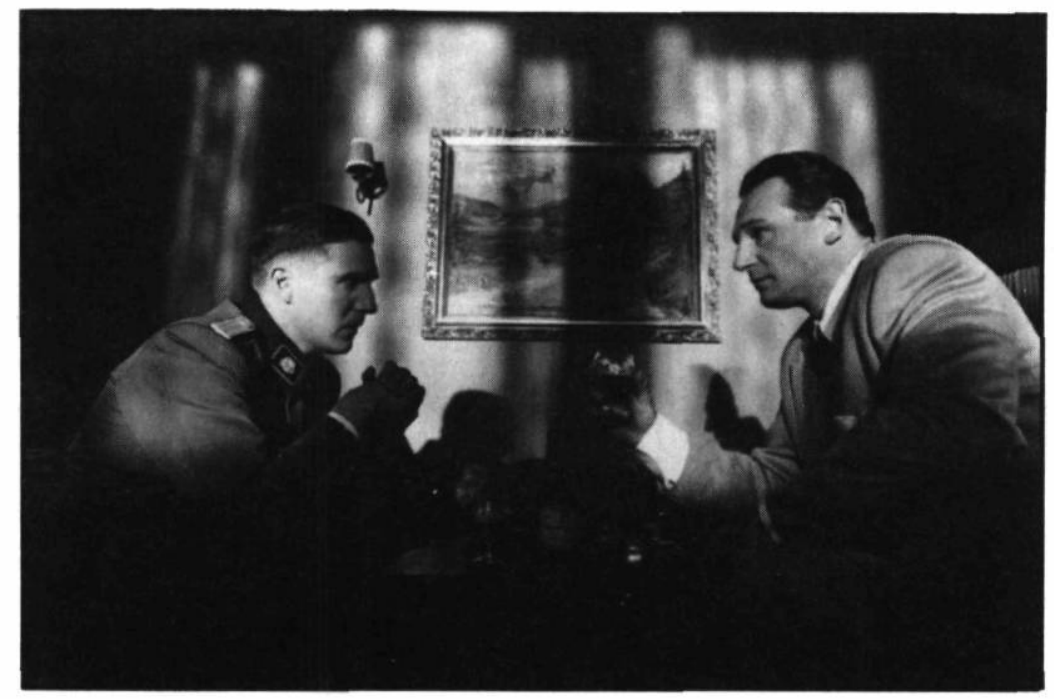

\section{Schindler's List de Steven Spielberg (1993)}

importantes sur les principaux acteurs du film et sur ce que le document nomme filmmakers, Spielberg mais aussi, presque au même titre, les divers producteurs du film de même qu'une note sur l'auteur du roman, Thomas Keneally, et sur sa source, Poldek Pfefferberg alias Leopold Page. Tous les principaux éléments du film sont réunis de façon à en rendre la publicité et la diffusion plus faciles. Le document précise que Spielberg a eu envie de faire ce film depuis la publication du livre de Keneally juste après la réalisation de $E$. T. en 1982, mais qu'il lui a fallu attendre 10 ans pour réaliser ce rêve. En somme, Schindler's List a toujours été un projet Spielberg. Dans ce document, la production comme le film sont trạités de façon narrative. La critique publique a tout ce qu'il faut pour faire son travail. En ce qui concerne la critique spécialisée et la critique universitaire, le problème se pose différemment. Le document d'accompagnement peut aussi faire l'objet d'une attention particulière et servir à l'élaboration d'un jugement critique plus solide. Il ne saurait toutefois remplacer de multiples visionnements du film ou même une recherche autour du phénomène qu'il représente.

Une première recherche parallèle, à partir des affirmations du document d'accompagnement, montre que Schindler's List n'est 
pas le projet poursuivi sans répit par Spielberg. Les droits du roman ont d'abord été achetés par Universal qui voulait produire une mini-série télévisée. Keneally en a même fait le scénario. Puis, Kurt Luedtke, scénariste de Out of Africa, a tenté de tirer du roman, mais sans succès, un scénario de long métrage. Spielberg, devant cette impossibilité, n'a plus manifesté d'intérêt pour le projet qui est alors passé dans les mains de Scorsese et du scénariste Zaillian. Après le retrait de Scorsese, Spielberg a enfin repris le projet et l'a mené à terme. Entre-temps, il a réalisé Twilight Zone-The Movie, Indiana Jones and the Temple of Doom, The Color Purple, Empire of the Sun, Indiana Jones and the Last Crusade, Always, Hook et Jurassic Park, soit 8 films en 10 ans... ce qui ne laisse pas beaucoup de temps pour se pencher sur les subtilités de l'Holocauste et le cas Schindler. C'est donc avec un regard légèrement différent que l'on peut aborder Schindler's List, un projet certes, une passion? Rien ne le prouve.

Le contenu du document et un visionnement du film sont suffisants pour former une critique publique avec ses pour et ses contre. On aura aimé ou non le traitement du sujet, le choix des comédiens, etc. La critique spécialisée et universitaire a besoin, elle, de comprendre les conditions de production d'un film pour en juger l'effet soit dans la carrière du réalisateur, soit dans l'histoire du cinéma. La distance est essentielle dans l'élaboration d'un jugement. Aussi, le contexte du film et de sa production acquiert une importance particulière. La question est ici d'autant plus complexe que l'Holocauste est un sujet éminemment tragique et que l'ensemble des films de Spielberg ne va pas du tout dans la veine de l'exploration du tragique. Une question se pose: comment réconcilier dans l'œuvre de Spielberg des films aussi différents que ceux qu'il a jusque-là réalisés et une volonté de mettre en scène, au moins partiellement, l'Holocauste? Andrew Sarris, cité par Douglas Brode, écrit en 1982 à propos de $E$. $T$ :

There is still too much of the world between the children's room and outer space left unexplored in the cinema of Steven Spielberg. He does not have to remake The Grapes of Wrath. All I ask is that sometime before he reaches the age of fifty, he should become 
somewhat more skeptical of his own self-induced euphoria (p. 226).

Schindler's List n'apparaît pas comme un choix naturel. Les indications fournies par le dossier Universal sur le tournage du film permettent de comprendre ultérieurement un certain nombre de choses qui demeurent inexplicables si on ne connaît pas ces détails qui sont fournis moins pour produire une analyse plus sophistiquée que pour montrer jusqu'à quel point Hollywood peut, s'il le faut, créer des conditions inégalées pour la production d'un film. Ces notes fournissent un résumé de l'histoire complètement centré sur le personnage d'Oskar Schindler et sa cohorte de Juifs, la fameuse liste de Schindler. En fait, ici, c'est à la fois le problème du roman de Keneally et celui du scénario de Zaillian qui se pose par rapport à la représentation même de l'Holocauste: comment raconter au cinéma ce qui n'est pas vraiment une fiction dans le roman. Le document d'accompagnement se présente comme un mode de lecture et cherche à gommer les difficultés que nous serons amenés à relever en citant en exergue une phrase du Talmud: "Whoever Saves One Life, Saves the World Entire." En somme, s'il n'y avait eu qu'un seul survivant, Hollywood aurait eu raison d'utiliser son exemplarité.

Les prix obtenus, l'approbation du film par la communauté juive et tout particulièrement par les survivants des Schindlerjuden, la reconnaissance presque universelle ont fait de ce film et de son réalisateur des scripteurs importants de l'histoire contemporaine. On pourrait comprendre que tout est alors dit et que le concert d'éloges et les critiques positives ont érigé ce film en monument à la mémoire de l'Holocauste et qu'il n'est pas très utile de vouloir y ajouter quelque chose. Pourtant, je vais tenter de montrer que, si la critique publique peut se contenter d'un discours homogène fondé sur une mise en contexte du film par la maison de production, il devrait en être tout à fait autrement des critiques plus spécialisées, qui tiennent compte des divers aspects de l'œuvre. Une telle entreprise pourrait illustrer comment la promotion du souvenir de l'Holocauste dans le cadre du cinéma hollywoodien est une entreprise risquée en fonction même du but poursuivi. 
Au regard d'une critique universitaire, Schindler's List pose des problèmes à trois niveaux: le récit filmique, les choix esthétiques, l'imaginaire. Le niveau le plus simple est certainement celui qui concerne l'histoire, la diégèse, au sens de narration. Qu'est-ce que le film raconte? Même si le titre du film comme celui du roman porte plutôt sur la liste des Juifs protégés par Schindler, le récit s'établit surtout autour des faits et gestes de la vie d'Oskar Schindler, Allemand sudète, membre du parti nazi, joueur, séducteur, profiteur, opportuniste, chevalier d'industrie, qui s'empresse de profiter de la situation faite aux Juifs de Pologne pour s'emparer d'une industrie qui appartenait à l'un d'eux et se faire une fortune en exploitant non seulement la maind'œuvre juive des camps de concentration mais aussi leur volonté de survivre à n'importe quel prix. Dans le film de Spielberg, Schindler est un personnage qui, contrairement à tous les autres, crève l'écran par sa présence, son charme, sa séduction. Il est difficile de ne pas retrouver chez ce personnage élégant des allures d'Indiana Jones ${ }^{4}$. La conversion tardive de Schindler à la cause des Juifs peut se comprendre comme un grand moment de conscience, une conversion, mais peut-être aussi comme la dernière carte que Schindler tente de jouer. Ses Juifs seront son sauf-conduit hors des territoires occupés par les Allemands et auprès des Américains avec lesquels il s'empressera de collaborer. Il est le héros principal du film, le sauveur volontaire ou involontaire sur lequel repose toute la commémoration spielbergienne de l'Holocauste. L'acteur Liam Neeson n'est pas allemand, mais il exprime fortement le caractère aryen du projet hitlérien. Son personnage est très près de celui que décrit Keneally dans le prologue de son roman. Cette partie du texte a beaucoup inspiré Zaillian et Spielberg dans leur mise en scène du personnage. L'ensemble du film le décrit comme un aventurier sans grande conscience morale si l'on entend par là une forme d'habitude de vie. Il règle les problèmes à mesure qu'ils se présentent et prend toujours bien soin de ne pas demeurer coincé dans une situation. Même dans le déménagement de son industrie d'Emalia à Brinnlitz en octobre 1944, on peut retrouver un désir de continuer à tirer au moins profit de la tranquillité au moment où la victoire bascule. Il est difficile de croire 
que Schindler ne se rendait pas compte de ce qui était en train de se passer sur le front. Lui, grâce à sa fausse usine, à ses Juifs et à l'argent, était à l'abri, au moins temporairement. Le personnage de Schindler est très individualisé si on le compare à cette espèce de foule anonyme que forment les Juifs à son emploi. Stern, joué par Ben Kingsley, est terne, porte-parole muet de la multitude des internés de race juive. $\mathrm{Si}$, comme l'ont fait remarquer plusieurs critiques au moment de la sortie du film, les Juifs sont représentés par les grandes masses, par les familles que l'on cherche à séparer et que les Juifs cherchent à maintenir, la personnalité individuelle est moins focalisée que dans le cas des principaux protagonistes allemands. En outre, la motivation des Juifs lorsqu'il s'agit de bouger à l'intérieur du système dans lequel les nazis les ont maintenus est la survie à n'importe quel prix, y compris le pot-de-vin exigé par des Juifs collaborateurs des Allemands. La constitution de la liste de Schindler - étaitce vraiment à la machine à écrire ? - a l'air de relever de l'improvisation, de la protection d'un petit cercle. Stern ne semble pas avoir beaucoup d'états d'âme. À la fin du film, dans la scène de départ inventée par Spielberg, il va même jusqu’à consoler Schindler en lui disant qu'il ne pouvait faire plus ou mieux. La réaction négative attendue du spectateur sera canalisée vers le personnage d'Amon Goeth, le commandant du camp de concentration de Plaszow, l'antagoniste "objectif" de Schindler. Le commandant est l'Allemand mauvais, sans nuances. Rien chez lui ne peut nous faire penser qu'il possède une quelconque qualité, un trait de caractère qui ne soit pas complètement noir. Il est psychotique et le film s'emploie à déployer les diverses facettes de son tempérament malade. La première fois qu'on le voit, une cinquantaine de minutes après le début du film, il arrive en voiture décapotable sur le site du camp de concentration, un mouchoir sur la figure moitié par dégoût pour les odeurs nauséabondes et moitié pour cause de rhume. On le verra ensuite en tueur fou, en alcoolique, en abuseur de femmes, en escroc. Plusieurs scènes le mettent en relation avec le seul Schindler. L'éclairage, qui favorise toujours ce dernier, la mise en scène de son élégance donnent l'impression d'une rencontre entre le bien et le mal, les deux étant campés de 
façon à s'exclure mutuellement. Cette composition permet de passer par-dessus les ombres du propre personnage de Schindler, ombres qu'avait signalées Keneally en lui attribuant le complexe Jekyll-Hyde. Cette nuance se perd dans le film. Schindler est placé dans des situations où n'importe quel spectateur sera tenu de lui accorder un maximum d'intentions droites, de preuves de courage, d'intelligence et d'habileté. Qu'il soit un coureur de jupons, quelqu'un qui supporte admirablement bien l'alcool, un aventurier riche toujours élégamment vêtu, cela, en fin de compte, joue plutôt en faveur de son personnage mis en valeur par l'opposition au personnage caricatural de Goeth.

Il est important de relever ici deux curiosités du scénario tourné. L'une porte justement sur Goeth. En 1944, il est luimême arrêté et mis en prison par les SS pour avoir exercé de la cruauté dans l'exercice de ses fonctions. Il en sortira complètement détruit mentalement et physiquement - il a alors le diabète - et voyagera jusqu'à Brinnlitz pour visiter Schindler. Il repartira sans qu'on sache très bien ce qu'il était venu faire à l'usine: retrouver un vieux complice ou quoi? Lautre concerne la scène finale où les prisonniers assistent, en pleurs, au départ de leur sauveteur: elle n'a jamais existé selon le témoignage même de plusieurs rescapés. Dans le roman de Keneally avec lequel, de façon générale, Spielberg ne prend pas trop de distances, la scène du départ est construite de façon fort différente. Schindler et sa femme, revêtus d'uniformes de prisonniers dans une Mercedez bourrée de diamants, se feront accompagner de huit prisonniers pour faire meilleure figure. Schindler emportera avec lui, outre la bague qui fait l'objet d'une mise en scène élaborée dans le film, une lettre en hébreu signée par trois prisonniers témoignant de la bonne conduite d'Oskar à leur endroit. Pressé de fuir, le convoi de Schindler suivra d'abord un convoi de la Wehrmacht avant de se diriger ensuite seul vers la Suisse. Il y parviendra grâce aux Américains d'abord et aux Français ensuite, alors qu'il se promène toujours avec sa "famille" juive qui lui sert en fait de caution. Il sera enfin libéré de toute accusation pouvant peser sur lui et sera protégé le reste de sa vie par les rescapés de Brinnlitz. On remarquera que ces deux écarts du film par rapport au roman vont dans le sens d'une évaluation 
positive du personnage de Schindler et d'une concentration sur lui du capital d'empathie du spectateur au mépris de ce qui se présente comme la vérité des témoignages juifs recueillis par Keneally au moment de l'écriture de son texte. Dans un traitement un peu plus subtil des indications du roman, le spectateur assiste à l'exécution par pendaison d'Amon Goeth qui a lieu, après procès, en Pologne. Au moment de la fuite de Schindler, cette exécution prend tout son sens: la disparition du mal va s'opposer au monument funéraire de Schindler en Israël, qui représente le bien dans toute son éternité. Tout est simple. Le bien est bien et le mal est mal. C'est dire que la diégèse retenue par le réalisateur et son scénariste s'est employée à grossir les traits jusqu'à la caricature de façon à empêcher l'existence de quelque doute que ce soit dans l'esprit du spectateur sur l'ambiguïté du personnage de Schindler. Ces remarques ne sont possibles que si la critique peut comparer des données avant de porter un jugement sur ce que le film met en scène et sur l'état de soumission auquel le spectateur est lui-même restreint.

Pourtant, Spielberg et son scénariste ont senti le besoin d'inventer un artefact de mise en scène qui puisse donner l'impression d'une certaine conscience morale chez Schindler. Ils l'ont trouvé dans la petite fille en rouge du film. Au moment de la liquidation du ghetto de Krakov à laquelle assiste Schindler à cheval et en costume d'équitation, une petite fille apparaît tout au loin dans la foule du ghetto et passe, insensible à la catastrophe qui l'entoure, à travers les masses de Juifs et les escadrons de soldats allemands. Elle marche à contre-courant du mouvement des diverses séquences pendant lesquelles on la suit. Dans le noir et blanc du film, elle est aisément repérable et le point de vue de la réalisation est celui de Schindler. La marche de la petite fille au manteau rouge dure environ deux minutes. À la fin de son périple, elle s'engouffre dans une maison abandonnée du ghetto. À partir de ce moment, Schindler ne peut évidemment plus la voir. Elle ira se cacher sous un lit et, à ce moment, elle redevient un personnage en noir et blanc. Plus tard, au moment où Goeth doit faire incinérer les cadavres des Juifs enterrés dans des fosses communes, on voit un très court flash: dans une charette où des morts sont empilés se trouve un petit cadavre tou- 
jours vêtu de rouge. Schindler, qui assiste, le voit aussi. Cet artefact est aussi très symptomatique de l'ambiguïté du film. Cette invention du scénariste Zaillian deviendra un trait distinctif de Schindler's List tout en constituant la citation d'un autre film. J'ai écrit plus haut la "petite fille au manteau rouge", mais j'aurais tout aussi bien pu écrire la "petite fille au manteau rose" puisque l'allusion ici se fait à partir de Rosebud dans Citizen Kane. C'est en effet le petit traîneau, symbole du souvenir et de l'enfance du tyran Kane, qui donne à Zaillian et Spielberg l'envie de répéter le même schéma. Le spectateur doit comprendre par cette intrusion de la couleur, ce marquage, que ce topos devient pour Schindler un lieu de conscience, de profondeur morale qui va lui permettre de se mettre d'une certaine façon au service de la cause juive. Du moins, c'est ainsi que le présente Brode:

Most important, Zaillian found a way to lick what Luedtke had considered the formidable "problem" posed by the Schindler story: Why Schindler did what he did. Luedtke had felt the need to understand, then dramatize, the essential reason for Schindler's transformation from card-carrying Nazi to El Cid of the Holocaust. This was not to be found in Keneally's book, which documented in detail what the man did without speculating about motivation. Luedtke had toyed with the idea of inventing dramatic scenes to make Schindler's psychology clear, but that struck him as melodramatic. Without this, Schindler's List would remain a docudrama, showing the surface of events, rather than allowing the audience a comprehension of them. Zaillian, on the other hand, proposed a "Rosebud" theory, drawing the phrase from one of Spielberg's favorite films, Citizen Kane, referring to the title character's final word. Kane's childhood sled is an "open" symbol, suggesting different things to different viewers. Zaillian's invocation of "Rosebud" had an immediate impact on Spielberg, who, lest we forget, is today the proud owner of the last remaining Rosebud sled prepared for that 1941 classic (p. 230-231).

Mais, comme on peut le voir par cette longue citation, c'est une figure multiple qui se pose devant nos yeux, un souvenir de Citizen Kane, un souvenir matériel appartenant à Spielberg, le 
souvenir d'une période, 1941, qui est englobée dans celle de l'Holocauste qui n'arrive pas à se dire à l'extérieur du lieu où le drame se perpètre 5 . On peut facilement établir d'autres ressemblances entre Kane et Schindler, deux personnages controversés, certainement mus par un désir de puissance, qui ne s'embarrassent pas plus qu'il ne faut de considérations éthiques. On peut aussi se poser des questions sur la nécessité de dramatiser ce qui ne l'est pas, c'est-à-dire la psychologie de Schindler. Et là, le cinéma hollywoodien annonce aussi sa couleur: l'absence d'intérêt pour ce qui ne serait qu'un docudrame qu'un spectateur ordinaire ne saurait comment interpréter. Dans le cinéma hollywoodien, le message existe, il doit être affiché, en rouge s'il le faut, et enrobé d'une histoire. C'est ça la véritable profondeur des choses. On peut mesurer ici la distance qui sépare Schindler's List des 14 heures du film de Jacques Lanzmann, Shoah!

$\mathrm{Si}$, dans le cadre de la culture filmique hollywoodienne, il doit y avoir une histoire, celle-ci ne peut être comprise qu'identifiée à un genre précis. Le film de Spielberg ne manque pas à la règle même s'il la complique un peu. Schindler's List représente un amalgame de genres: le film d'aventures si l'on regarde la trame narrative qui suit Schindler lui-même, le film de guerre si l'on pense surtout aux scènes entourant les camps de concentration et le personnage de Goeth. Le sort des Juifs est, lui, traité comme un matériau obligatoire. On s'assure qu'il se trouve mis en place dès le début, avec l'ouverture en couleurs sur la présence de la tradition juive, puis sur les deux bougies. La couleur se retrouvera dans l'usine d'Oskar lors d'une cérémonie célébrée par le rabbin puis, à la toute fin du film, au moment où les rescapés de la liste de Schindler se retrouvent en Israël alors qu'ils étaient en marche, dans la section en noir et blanc, en direction d'une petite ville dans les environs de leur dernier camp de travail à Brinnlitz. L'ensemble a des airs de saga qui ressemblent à tous ces films décrivant les rapports avec la frontière. Les critiques n'ont pas manqué de relever les rapports de Schindler's List avec les autres films de Spielberg, leurs aventures comme celles qui attendent toujours Indiana Jones, leurs autres mondes comme dans Close Encounters of the Third Kind et E. T. Les ressemblances sont parfois étonnantes. Cela amènera certains cri- 
tiques à préciser que Schindler's List est bien un film de Spielberg comme tous ces autres films et moins un point de vue sur l'Holocauste. Nous suivons ici l'histoire d'un homme, d'un aventurier, à la fois Jekyll et Hyde, qui sauve vraisemblablement environ 1000 Juifs de la mort dans une catastrophe de plus de 6000000 de cadavres mais qui, au-delà de toute prévision, se sauve lui-même, porté par la reconnaissance mais peut-être aussi par la culpabilité de certains des rescapés.

La critique narratologique peut aider le point de vue de la critique publique à s'approfondir en faisant ressortir des éléments narratifs qui vont dans le sens de tel ou tel aspect des données présentées à l'écran. Mais cela ne suffit pas. Il faut aussi pouvoir aborder le traitement du film, les moyens utilisés par le film. Ici, le dossier de presse de Schindler's List, toujours lui, est particulièrement utile. Il présente en fait certaines données sur la fabrication du film comme la décision de tourner en noir et blanc, de tourner aussi en Pologne mais à l'extérieur des camps dont il a fallu reconstruire des sections entières, étrange duplication, d'utiliser la caméra à l'épaule pour donner au film son allure documentaire, de recourir à divers procédés d'éclairage et de couleurs pour faciliter un certain type de film noir et blanc. Ces éléments sont à la fois techniques et esthétiques. On peut leur ajouter les angles de prise de vue lorsque le sujet montré fait partie du récit consacré à Oskar Schindler ou à Amon Goeth, par exemple, plutôt que lorsqu'il illustre la vie et le sort des Juifs polonais.

La perspective technique et esthétique de Spielberg est, en quelque sorte, opposée au traitement narratologique qu'il a choisi : donner l'impression d'un documentaire sur le plan du matériau. Cela ne peut être une décision purement esthétique. Elle s'ouvre sur l'imaginaire, sur ce que représente l'archive comme matériau authentique d'un documentaire. L'Holocauste a ses propres archives, insoutenables, dernière trace d'une vérité qui a échappé si longtemps à la conscience des peuples et des gouvernements et qui demeure, encore aujourd'hui, avec les lieux historiques, les meilleurs moyens de pénétrer cet univers si difficile à comprendre tant par son horreur que par son absurdité. Que peut vouloir dire alors cette volonté de "mimer" les 
archives, de libérer les caméras pour garantir une certaine forme de vérité, de faire avec les caméras ce qu'ont fait les Allemands en train de documenter, pour l'histoire, la solution finale? Peuton mettre sur le même pied un film qui se veut de style néo-réaliste italien et des archives dont la matière même est un défi à toute tentative de fiction? On sait que, si la fiction peut se donner des airs de réalité, elle finit toujours par se mettre elle-même en question au profit d'un intérêt pour la référence. À la toute fin du film, quand les comédiens et comédiennes accompagnent les survivants de la liste de Schindler, on ne sait s'il faut rire ou pleurer. C'est la confirmation de la fiction, dans un geste mélodramatique qui l'emporte sur celle d'une quelconque recherche de vérité. Il est plus facile de dramatiser que de comprendre. Or la dramatisation est aussi une forme de banalisation. Le cinéma d'Hollywood défend une culture du spectacle, de la mémoire du spectacle et non de prétendus faits qui auraient pu être à leur origine. C'est le même débat que suscite J. F. K. d'Oliver Stone qui a choisi, en apparence du moins, l'archive mais pour mieux la vider de tout contenu de vérité en cherchant, à la manière d'un enquêteur, à retrouver ailleurs la "vraie vérité", dans une sorte de roman-plaidoyer. On doit aller encore plus loin dans la critique des procédés de Spielberg. On ne monte pas une série d'archives. On les place en contiguïté en choisissant un ordre qui s'impose, la chronologie, la description, etc., et un ordre qui impose le respect. En faisant du noir et blanc de fiction pour représenter certains moments de l'Holocauste, Spielberg choisit de donner une intensité dramatique à des gros plans. C'est vraisemblablement pour cette raison qu'il n'est pas donné de voir la déchéance physique des corps et, à la limite, de l'oublier, puisque l'on se trouve constamment en présence d'acteurs et d'actrices qui ont l'air bien nourris. L'absence d'expression dans les figures émaciées des archives est remplacée par une émotion mise en scène, travaillée par l'éclairage, rendue efficace par le montage ou encore le point de vue de la caméra. Ce qui est plus grave encore, c'est le traitement de Schindler et des autres nazis comme des documents d'archives. Il existe peut-être quelque part des archives allemandes témoignant de la vie militaire pendant la seconde guerre mondiale. Pourrait-on les mettre en con- 
tiguïté avec les archives de l'Holocauste? C'est pourtant ce qui est, ici, donné à voir: une banalisation de l'archive.

Une critique qui connaît tous les ressorts du cinéma moderne ne peut laisser de côté des éléments aussi importants. Schindler's List est un spectacle, l'Holocauste ne l'est pas. Il y a des sujets face auxquels il vaut mieux baisser les yeux, de dégoût, de honte, d'impuissance, que de se laisser porter par le rythme d'un montage efficace au sens hollywoodien du terme, c'est-à-dire d'un spectacle qui n'ennuie pas.

Ces diverses considérations ouvrent tout naturellement sur la question de l'imaginaire. Que retiendra-t-on de l'Holocauste dans 50 ans? La vision de Spielberg? Un massacre dont certains doutent et, en avant-plan, une véritable épopée hollywoodienne avec un sauveur et des sauvés? Des millions de Juifs exterminés par des Allemands qui obéissent aveuglément aux ordres d'Hitler et un millier de Juifs sauvés par un Allemand obéissant souvent mais pas toujours aux ordres d'Hitler? L'histoire d'un homme exceptionnel qui s'est battu contre le pouvoir dans son pays pour libérer des Juifs? Un épris de justice? La question de ce qui se donne à voir est primordiale, car c'est la seule chose en bout de compte que l'on retiendra. Or, c'est justement l'ambiguïté du film de Spielberg qui peut avoir un effet aseptisant sur le spectateur, une mise à distance qui lui permet de se dire que tout n'était pas aussi noir qu'on a bien voulu le dire. Il existe toujours des bons et des méchants et, dans la mesure du possible, faciles à distinguer les uns des autres. La "vérité" du film de Spielberg vaudra bien la vérité des archives. La critique doit obligatoirement se pencher sur l'effet idéologique des images qui hantent la culture cinématographique en général et la culture hollywoodienne en particulier. Schindler's List n'est ni le premier ni le dernier film sur le sujet de l'Holocauste, mais la célébration qui l'a entouré depuis sa mise en diffusion cadre mal avec l'Histoire, celle qui ne se revit pas deux fois - du moins on l'espère —, celle qui a beaucoup de difficulté à se dire, à s'installer dans la mémoire de chacun en y engageant autre chose que le plaisir d'avoir vu un bon film, un film bien fait dont on ira discuter chez Schwartz ou chez Moshe.

C'est la réflexion hollywoodienne sur la vie, sur la mort qu'il faut avoir le courage de dénoncer à travers le film de Spielberg. 
C'est aussi l'effet de mémoire collective que cette culture fournit à une très grande partie de l'humanité. À la toute fin du film, des roses sont placées sur la tombe de Schindler par un bras qui appartient à un corps que l'on ne voit pas. Dans le plan suivant, de très loin, on voit un homme se tenir tout près de la tombe de Schindler. Il faut que cet homme soit Spielberg, venant signer ici sa version de l'horreur de l'Holocauste. Il a choisi la tombe d'un Allemand, d'un sauveur très controversé dont l'épouse, Emily Schindler, publie ces jours-ci ses mémoires dans lesquels elle prétend que la liste de Schindler tapée et bien faite n'a rien à voir avec Oskar, son illustre mari. On retrouvera sans doute bien d'autres raisons de se questionner quand ce texte sera, lui aussi, disponible.

On comprend que la critique publique ne puisse élaborer tous ces points de vue et plusieurs autres au moment de la sortie d'un film. Elle en serait tout à fait incapable. Mais il faut comprendre qu'une fois qu'elle s'est prononcée, la critique publique perd son droit de parole et le film, lui, continue son chemin dans l'imaginaire des collectivités auxquelles il est présenté. La critique spécialisée doit chercher, par tous les moyens, à constituer son propre discours, son droit de parole, à l'imposer, alors que les films échappent de plus en plus au jugement critique des spectateurs. Si le film a plusieurs vies selon ses moyens de diffusion, il faudrait que la critique puisse connaître le même sort et ne pas devenir, avec le temps, une cote numérique ou un visa de censure qui se contente de discriminer les publics selon l'âge. Savoir comment un film est fait, c'est aussi en connaître le travail sur les imaginaires collectifs.

Université du Québec à Montréal

\section{NOTES}

1 J'exagère un peu, mais si peu! Peut-être le cinéma pourra-t-il se retrouver lorsqu'il sera repris par des amateurs.

2 On a actuellement sur le marché des technologies sans objet! La télévision à haute définition n'est toujours pas disponible et le son surround n'est codé que dans une minorité de vidéocassettes. Le consommateur ne se plaint pas, car il ne peut faire la différence.

3 Cela a un intérêt puisqu'en le comparant avec les cotes octroyées dans d'autres répertoires, on retrouve généralement la même appréciation. 
4 "A reporter for the L.A. Times/Calendar, visiting the set, recalls approaching Liam Neeson, standing near a period car with white running boards, looking absolutely dapper in a beautifully cut suit and white-brimmed fedora. Before the journalist could even utter a word about Neeson's striking resemblance to Harrison Ford in Spielberg's trilogy, Neeson beat him to the punch: "No Indiana Jones jokes, okay?" " (Brode, p. 235).

5 Dans un autre moment crucial du film, lorsque les femmes sont amenées par erreur à Auschwitz et qu'elles sont rasées, mises à nu et forcées d'entrer dans une des fameuses salles de douches, il y a une évocation de la fameuse scène de douche de Hitchcock dans Psycho. Cette fois-ci, miracle, l'eau remplace le gaz et signifie la vie.

\section{OUVRAGES CITÉS}

Brecher, Elinor J. "Schindler's Legacy", True Stories of the List Survivors. New York: Penguin Books, 1994.

Brode, Douglas. The Films of Steven Spielberg. New York: A Citadel Press Book, 1995.

Keneally, Thomas. La liste de Schindler. Paris: Éditions J'ai lu, 1994.

Leventhal, Robert S. [Department of German, University of Virginia]. Romancing the Holocaust, or Hollywood and Horror: Steven Spielberg' Schindler's List. Internet : 1995.

"Schindler's List ", Production Information (dossier de presse). Los Angeles: Universal City Studio and Amblin Entertainment, 1995. 